\title{
XLIX. On certain sources of error in connection with experiments on torsional vibrations
}

\section{Herbert Tomlinson B.A.}

To cite this article: Herbert Tomlinson B.A. (1886) XLIX. On certain sources of error in connection with experiments on torsional vibrations, Philosophical Magazine Series 5, 22:138, 414-419, DOI: $10.1080 / 14786448608627955$

To link to this article: http://dx.doi.org/10.1080/14786448608627955

册 Published online: 29 Apr 2009.

Submit your article to this journal $[\pi$

Џ Article views: 2

Q View related articles $₫$ 


\section{$414 \mathrm{Mr}$. H. Tomlinson on certain Sources of Error in}

circuit was broken, and the plates taken out of the liquid. The cathode was found to be covered with a good deposit of copper, but the anode was completely enclosed in a glacial sheath of copper sulphate.

Another cell, in which the anode had a surface of 4.4 square centimetres, was treated in the same way, and was found to carry a current of one-tenth of an ampere for 14 minutes. The plates were then found to be covered by a similar coating of copper sulphate.

A similar cell in which the anode had a surface of 7 square centimetres was found to carry one-tenth of an ampere for 30 minutes, when the current fell off as before from the same cause.

Experiments were then made in a similar manner with cells containing a solution the density of which was $1 \cdot 11$. When the current density at the anode exceeded one ampere per 20 square centimetres, the plate became covered with black oxide and the current diminished greatly in strength after a few minutes (about 7 minutes for a current-density of one ampere to 7 square centimetres, and 3 minutes for double that current-density). The current does not entirely cease, and will after a few minutes, if the battery be of sufficiently high potential, again assume nearly its former strength. The oxide then falls off and gases are liberated at the surface of the anode, forming a descending stream close to the plate and an ascending stream two or three millimetres further out. As the current-density is diminished, less and less oxidation takes place and it becomes a lighter brown in colour. With a current-density of one ampere to 30 square centimetres the anode became covered with brown oxide, which made the resistance of the cell high and variable, but little or no gas was generated.

No gas was, so far as could be observed, given off at the cathode during any of these experiments.

XLIX. On certain Sourees of Error in Connection with Experiments on Torsional Vibrations. By Hereert TomLINsoN, B.A.*

\section{Introduction.}

DURING a long series of researches on the torsional elasticity and internal friction of metals, I have come across certain sources of error in connection with torsional vibrations

- Communicated by the Physical Society : read June 26, 1886. 
generally, which I venture to bring before the notice of the Physical Society in the hope that by so doing I may perchance save others who may pursue similar investigations from the pitfalls into which I have fallen, and from which I have managed to extricate myself only after a considerable expenditure of time and labour.

In my earlier experiments a wire about 600 centims. in length and 1 millim. in diameter was suspended vertically, having its upper extremity clamped to a rigid support and its lower one clamped or soldered to the centre of a horizontal bar of brass, from which were suspended, by threads or fine wires, two cylinders of equal dimensions and mass, and placed at equal distances from the wire. The torsional oscillations of the wire were observed by means of the usual arrangement of mirror, scale, and lamp, the main object of the inquiry being to determine as accurately as possible the logarithmic decrement of the amplitude of swing and the vibration-period. After one set of observations had been completed, the moment of inertia was altered by sliding the cylinders along the bar further away from or nearer to the wire, so that the latter might now vibrate in a different period. If $t$ be the period of vibration, $k$ the moment of inertia, and $f$ the torsional couple,

$$
f=\frac{\pi^{2} k}{t^{2}}
$$

The moment of inertia of the bar together with that of the suspended cylinders could be calculated with very fair accuracy; the value of $t$ was also calculated to a nicety; so that it was reasonable to expect that the values of $f$ obtained with different moments of inertia should be very fairly in accordance with each other. For some time this proved to be the case, and several wires of different metals had already been examined when a curious phenomenon presented itself. A change of moment of inertia had just been made, and the wire was then set in torsional oscillation; but instead of the amplitude of swing diminishing by slow degrees as in the previous experiments, the spot of light was seen to make five or six oscillations of very rapidly diminishing amplitude, and finally come nearly to rest. Suon, however, the amplitude began to increase until in a few vibrations it extended over some two hundred divisions of the scale, when once more diminution and, finally, rest nearly ensued. I will not trouble the Society with my conjectures at the time as to the cause of the phenomenon ; suffice it that at length I discovered, what perhaps I ought to have discovered at once, that the, at first sight, startling apparition was due to a very natural cause, 
namely, the rotation to and fro of the cylinders about their axes, and that the rapid absorption of energy was owing to the fact that the torsional vibration-period of the wire nearly synchronized with the vibration-period of the cylinders about their uxes. I need hardly say that after this discovery I considered it necessary to re-try all my previous experiments with improved arrangements. With more perfect apparatus, in which the cylinders were clamped to the bar so as to be incapable of motion independently of the latter, I was glad to find the main conclusions which I had previously drawn respecting internal friction confirmed. On one occasion, however, by what afterwards turned out to be the merest chance in the world, the old phenomenon appeared with all its curious concomitants, and beats were very plainly discernible between two sets of vibrations which nearly synchronized. These two sets of vibrations I set to work to disentangle in a manner kindly suggested to me by Prof. G. G. Stokes, with whom I had some correspondence on the subject. Suppose that in one or more beat-intervals the slower vibration is dominant*, and afterwards the quicker. If the amplitudes cross during a beat-interval, that one is to be deemed dominant which is dominant about the time of lull. Suppose there are $m$ beatintervals in which the slower is dominant, followed by $n$ in which the quicker is dominant, and let $\mathrm{N}$ be the total number of vibrations counted $\dagger$. Then the number of vibrations of the two component kinds will be:-

For the slower, $\mathrm{N}-2 n$,

For the quicker, $\mathrm{N}+2 m$.

In this way I managed without any difficulty to find that one of the two vibration-periods was nearly a constant whatever the moment of inertia; whilst the other, that due to the torsional elasticity of the wire, increased as the square root of the moment of inertia. At first I felt very strongly inclined to believe that the former of the two vibration-periods pertained to some molecular state of the wire; and I was especially deceived by the fact that always after rest the phenomenon became less marked, and, finally, almost vanished, easily, however, to be reproduced in all its former intensity by the slightest shock given to the wire, or by raising or lowering the temperature slightly. The wire was of iron, and my previous experience of the effect of rest on the molecular dispo. sition of this metal led me to draw the above-mentioned conclusion. Had I not been thus deceived, I might more quickly

- By dominant vibration is meant the vibration of greater amplitude.

+ For accuracy, the counting should begin and end with a maximum. 
have arrived at the solution of the problem; but as it was four weeks were spent in endeavouring to find the effect of change of temperature, change of load, and change of mode of attaching the cylinders, until I discovered that the phenomenon was simply caused by approach to synchronism between the periods of torsional and pendulous vibrations of the wire. Of course, if the axis of the wire passed accurately through the centre of mass of the vibrator, the phenomenon would not occur, but it is impossible quite to secure this; and as a consequence so-called centrifugal force on the one hand, and the force of gravity on the other, set up pendulous vibrations which may seriously interfere with the vibration-period due to the torsional elasticity, and still more so with the logarithmic decrement of arc.

Having thus discovered two enemies in ambush, I thought that the ground was now clear, at least as far as any danger from synchronism was concerned; but in this I was mistaken: for happening to pursue my investigations still further with comparatively light loads and small moments of inertia, I at length became acquainted with a third source of error arising from approach to synchronism between the torsional and transverse vibrations of the wire. It is, I believe, quite impossible to excite torsional vibrations without at the same time exciting transverse ones; and should synchronism nearly occur between the periods of these vibrations, we may have beats quite as strongly marked as in the two cases before mentioned.

There is yet another source of error to which it is desirable to draw attention. When a wire has been recently suspended, the torsional vibration-period will always be found slightly greater than when it has been suspended for some time and frequently set in vibration. The length of time which must elapse before a wire has reached its state of maximum torsional elasticity depends upon the nature of the metal; with copper, for instance, a few hours will suffice, whilst iron may require at least a couple of days. Now suppose that this state of maximum elasticity has been reached: if we wish to preserve it, care must be taken not to jar the wire or to subject it to a change of temperature of even two or three degrees Centigrade; for in either case the elasticity will be slightly diminished, and a few hundred vibrations will be required to restore it to its former condition. Since it is very difficult to avoid such slight changes of temperature as those mentioned above, it is always advisable after any rest to cause the wire to vibrate a few hundred times before beginning fresh observations. 
Determinations of Torsional Electricity.

It is evident, from what has gone before, that when the cylinders are suspended from the bar by threads, the torsional elasticity, as inferred from the vibration-period, may be seriously affected should the torsional vibration-period of the wire approach to synchronism with the vibration-period of the cylinders about their axes; and to such an extent did I find this to be the case, that the values of the torsional couple for the same wire, as calculated from the previously given formula, differed from each other by 10 per cent. or more when the moment of inertia was varied.

The effect of synchronism between the torsional vibrations of the wire and the pendulous vibrations is not likely, if proper care be taken in the construction and disposition of the bar and its appendages, to be great; but it may nevertheless be very sensible.

Synchronism between the torsional and transverse vibration-periods is not likely to occur except in extreme cases; but it must be remembered that the torsional vibration-periods may be affected by synchronism between them and multiples of the transverse vibration-period, though of course not to the same extent as when the fundamental transverse vibrationperiod equals the torsional vibration-period. A similar remark to the above would also apply to the other two cases of synchronism.

\section{Determinations of Magnetic Moments.}

It is evident that determinations of magnetic monents by the method of oscillations may be similarly affected by the same causes as we have seen would affect determinations of the torsional elasticity.

\section{Damping of Magnets.}

Still greater error may be introduced from the above causes in experiments on the damping of vibrating magnets; for the logarithmic decrement is much more seriously affected than the vibration-period.

\section{Determinations of Moments of Inertia.}

When it is impossible to determine the moment of inertia of a body by measurement of its dimensions and mass, it is usual to find the time of oscillation, and afterwards to redetermine this time when the moment of inertia has been altered to a known extent.

If $t$ and $t^{\prime}$ be the two times of oscillation, $\mathrm{K}$ the required 
moment of inertia, and $k$ the known change of moment of inertia,

$$
\mathrm{K}=k \frac{t^{2}}{t^{t^{2}-t^{2}}} .
$$

Now a small error in the determination of either $t$ or $t^{\prime}$ produces a comparatively large error in the value of $K$; and it is therefore of considerable importance here that there should be no approach to the previously mentioned synchronisms. It is important also here to notice the fourth source of error which has been mentioned; for it is very difficult to change the moment of inertia without imparting some shock to the wire.

\section{On the Self-induction of Wires.-Part IV. By Oliver Heaviside*}

A S mentioned at the close of Part III., it would appear A that the only practicable way of making a workable system, which will allow us to introduce the terminal conditions that always occur in practice, in the form of linear differential equations connecting $\mathrm{C}$ and $\mathrm{V}$, the current and potential difference at the terminals, is to abolish the very small radial component of current in the conductors. This does not involve the abolition of the radial dielectric current which produces the electric displacement, or alter the equation of continuity to which the total current in the wires is subject. The dielectric current, which is $S \dot{V}$ per unit length of line, and which must be physically continuous with the radial current in the conductors at their boundaries, may, when the latter is abolished, be imagined to be joined on to that part of the longitudinal current in the conductors that goes out of existence by some secret method with which we are not concerned.

We assume, therefore, that the propagation of magnetic induction and electric current into the conductors takes place, at any part of the line, as if it were taking place in the same manner at the same moment at all parts (as when the dielectric displacement is ignored, making it only a question of inertia and resistance), instead of its being in different stages of progress at the same moment in different parts of the line. This requires that a small fraction of its length, along which the change in $\mathrm{C}$ is insensible, shall be a large multiple of the radius of the wire. The current may be widely different in strength at places distant, say, a mile, and yet the variation in a few yards be so small that this section, so far as the propa-

* Communicated by the Author. 